\title{
Pulsed intravenous corticosteroids in chronic inflammatory demyelinating polyneuropathy: why not?
}

\author{
Daniele Orsucci \\ Unit of Neurology, San Luca Hospital, Via Lippi-Francesconi, Lucca 55100, Italy. \\ Correspondence to: Dr. Daniele Orsucci, Unit of Neurology, San Luca Hospital, Via Lippi-Francesconi, Lucca 55100, Italy. \\ E-mail: orsuccid@gmail.com
}

How to cite this article: Orsucci D. Pulsed intravenous corticosteroids in chronic inflammatory demyelinating polyneuropathy: why not? Neuroimmuno/ Neuroinflammation 2019;6:3. http://dx.doi.org/10.20517/2347-8659.2019.05

Received: 11 Feb 2019 Accepted: 12 Feb 2019 Published: 24 Feb 2019

Science Editor: Athanassios P. Kyritsis Copy Editor: Cai-Hong Wang Production Editor: Huan-Liang Wu

Chronic inflammatory demyelinating polyneuropathy (CIDP) is a remitting/relapsing and/or chronic autoimmune disorder, characterized by symmetrical, sensorimotor neuropathic involvement and a slowly progressive onset. There are many clinical variants, suggesting that this disorder may not be an unique entity but rather a spectrum ${ }^{[1]}$. CIDP diagnostic criteria combine clinical and electrophysiological features. Supportive data include increased cerebrospinal fluid (CSF) protein levels ${ }^{[1]}$.

Many, but not all, patients may be successfully treated with therapies aimed at arresting immunological mechanisms, such as corticosteroids $s^{[2]}$ and intravenous immunoglobulins (IVIg) ${ }^{[3]}$. A systematic review concluded that there was no clear short-term difference with IVIg when compared with intravenous methylprednisolone and likely no improvement when compared with either oral prednisolone or plasma exchange $^{[4]}$. More randomised trials are strongly needed ${ }^{[4]}$.

Recently, a multicentre retrospective study compared safety and efficacy of daily prednisolone, pulsed dexamethasone, and pulsed intravenous methylprednisolone. Interestingly, corticosteroids led to improvement in $60 \%$ of subjects and to clinical remission in $61 \%$ of responders ${ }^{[2]}$. There were no significant differences in terms of safety and efficacy ${ }^{[2]}$. A therapeutic protocol with corticosteroids, with IVIg as an adjunctive treatment in case corticosteroid treatment was insufficient, could lead to improvement in $90 \%$ of CIDP patients ${ }^{[2]}$.

Some patients may not respond to IVIg. For instance, we follow a 37-year-old male patient diagnosed with CIDP at age 7, based on a sensory ataxic phenotype. The diagnosis was supported by typical

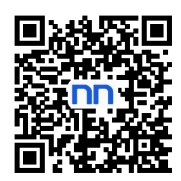


electrophysiological features, CSF analysis and sural nerve biopsy. Molecular studies for hereditary neuropathies were unremarkable. At age 33 he had a severe relapse leading to a subacute flaccid, areflexic tetraparesis unresponsive to IVIg. He was then successfully treated with plasma exchange and intravenous corticosteroids. Subsequently he became corticosteroid-dependent needing chronic treatment with oral prednisone (25 mg every other day). Unfortunately, he developed bilateral cataract, right hip osteonecrosis and cushingoid appearance. Therefore, we switched the treatment to pulsed intravenous methylprednisolone ( $1 \mathrm{~g}$ daily for three consecutive days every two months). After one year of this schedule, the neuropathy is excellently controlled (apart from mild distal leg weakness) and corticosteroid toxicity is minimized, with improvement of hip osteonecrosis and regression of the cushingoid features.

In conclusion, corticosteroids are cheaper, easier to use, and much more widely available than IVIg. They have been suggested to lead to long-term remission more often than $\operatorname{IVIg}^{[2]}$. Furthermore, even if there are no significant differences in response and remission rate between these two regimens, pulsed intravenous corticosteroids have lower rates of serious adverse effects than long-term daily use ${ }^{[2]}$. Therefore, in our opinion pulsed intravenous methylprednisolone should be considered in CIDP patients, especially in nonresponders to IVIg. In fact, it may represent the therapy of choice in these patients.

\section{DECLARATIONS}

\section{Authors' contributions}

Made substantial contributions to conception and design of the study and performed data analysis and interpretation: Orsucci D

\section{Availability of data and materials}

Not applicable.

\section{Financial support and sponsorship}

None.

\section{Conflicts of interest}

The author declared that there are no conflicts of interest.

\section{Ethical approval and consent to participate}

Not applicable.

\section{Consent for publication}

Not applicable.

\section{Copyright}

(c) The Author(s) 2019.

\section{REFERENCES}

1. Mathey EK, Park SB, Hughes RA, Pollard JD, Armati PJ, et al. Chronic inflammatory demyelinating polyradiculoneuropathy: from pathology to phenotype. J Neurol Neurosurg Psychiatry 2015;86:973-85.

2. van Lieverloo GGA, Peric S, Doneddu PE, Gallia F, Nikolic A, et al. Corticosteroids in chronic inflammatory demyelinating polyneuropathy: a retrospective, multicentre study, comparing efficacy and safety of daily prednisolone, pulsed dexamethasone, and pulsed intravenous methylprednisolone. J Neurol 2018;265:2052-9.

3. Kuwabara S, Mori M, Misawa S, Suzuki M, Nishiyama K, et al. Intravenous immunoglobulin for maintenance treatment of chronic inflammatory demyelinating polyneuropathy: a multicentre, open-label, 52-week phase III trial. J Neurol Neurosurg Psychiatry 2017;88:832-8.

4. Oaklander AL, Lunn MP, Hughes RA, van Schaik IN, Frost C, et al. Treatments for chronic inflammatory demyelinating polyradiculoneuropathy (CIDP): an overview of systematic reviews. Cochrane Database Syst Rev 2017;1:CD010369. 